\title{
Antipsychotic prescription to identify delirium: results from two cohorts
}

Kristin M Zimmerman'

Allison M Paquin ${ }^{2}$

James L Rudolph ${ }^{3,4}$

'Department of Pharmacotherapy and Outcomes Science, Virginia

Commonwealth University, Richmond,

VA, ${ }^{2}$ Connecticut Center for Primary

Care, ProHealth Physicians, Inc.

Farmington, CT, USA; ${ }^{3}$ Research and

Development Service, Center of Innovation in Long-Term Services and Supports, Providence VA Medical Center, ${ }^{4}$ Warren Alpert Medical School and Brown School of Public Health, Brown University, Providence, RI, USA
Correspondence: James L Rudolph Research and Development Service, Center of Innovation in Long-Term Services and Supports, Providence VA Medical Center,

860 Chalkstone Avenue, Providence, RI 02908, USA

Tel +l 40I 2737 I00 ext 6406

Fax + I 40I 45733 II

Email James.Rudolph@va.gov
This article was published in the following Dove Press journal:

Clinical Pharmacology:Advances and Applications

3 October 2017

Number of times this article has been viewed

Objectives: Detection of delirium in hospitalized patients remains challenging. The objective was to determine if the prescription of antipsychotic medications was associated with delirium.

Patients and methods: Two patient cohorts were utilized from a tertiary Veterans Affairs hospital: a palliative care retrospective cohort and a prospective medical cohort. Patients prescribed outpatient antipsychotics were excluded. Retrospectively, delirium was identified using a validated medical record-review instrument. Prospectively, a clinical expert assessed patients for delirium daily using a standardized interview. Acute antipsychotic medication administration was recorded from the electronic medical record.

Results: In the retrospective cohort $(n=217)$, delirium was found in $31 \%(n=67)$ and antipsychotic use in $18 \%(n=40)$ of patients. Acute antipsychotic use indicated delirium with $54 \%$ sensitivity and $97 \%$ specificity. In the prospective cohort $(n=100)$, delirium developed in $23 \%$ $(n=23)$ and antipsychotics were used in $5 \%(n=5)$ of patients. The sensitivity and specificity of acute antipsychotic use was $22 \%$ and $100 \%$, respectively.

Conclusion: Hospitalized patients who are acutely prescribed antipsychotics are likely to have delirium, but not all patients with delirium will be identified with this method. In health systems, utilization of the prescription of acute antipsychotics can be an efficient and specific method to identify delirious patients for targeted intervention.

Keywords: delirium antipsychotic aged, palliative care, quality improvement, pharmacy practice

\section{Background}

Acute fluctuations in cognition and attention and impairment in perception or consciousness associated with delirium result in significant increases in morbidity and mortality. ${ }^{1}$ Delirium occurs in $11 \%-42 \%$ of medical inpatients ${ }^{2}$ and $26 \%-62 \%$ of palliative care patients. ${ }^{3}$ Delirium is also associated with increased risk of short- and long-term mortality, medical complications, and prolonged length of stay, as well as residual functional impairment resulting in nursing home placement. ${ }^{1}$ Despite the tremendous morbidity and mortality, without standardized assessment, delirium is frequently missed. Research over the past 25 years has found that up to $75 \%$ of delirium will be not be reported in the medical record..$^{4-7}$ As a result, there is an urgent need to identify delirium for research and quality-improvement projects by hospital systems.

The gold-standard diagnosis of delirium - clinician interview followed by completion of diagnostic criteria - needs to be completed during hospitalization and requires substantial time (30-90 minutes). ${ }^{8}$ However, the time and training required make prospective case identification cost-prohibitive for quality-improvement programs and 
health systems where efficient identification is imperative. Retrospective case identification for delirium is also complex. Past work utilizing ICD9/10 codes or physician diagnoses for delirium found these to be inaccurate and returned low prevalence rates. ${ }^{9}$ Standardized case-identification tools require a trained person to conduct a thorough chart review for key delirium terms, which requires 10-25 minutes per chart. ${ }^{10}$ Therefore, there is a need to identify patients with delirium efficiently and effectively using rapid electronic methods.

Antipsychotics are first-line medications for managing agitation associated with delirium. ${ }^{11,12}$ Importantly, antipsychotics do not treat the underlying causes. The use of antipsychotics to control agitation is particularly important in those with the hyperactive delirium subtype, and in particular when the features of delirium are distressing to caregivers and family. While there is considerable debate about the use of antipsychotics in the prevention of delirium in high-risk patients, ${ }^{13-15}$ a new prescription for an antipsychotic medication in hospitalized patients may represent an underlying delirium.

The purpose of this study was to determine if new, in-hospital antipsychotic prescribing was associated with delirium. First, in a retrospective cohort, we examined if new prescription of an antipsychotic was associated with delirium. An additional validation step utilized a prospective cohort to determine if new antipsychotic prescription was associated with delirium.

\section{Patients and methods}

These studies were in accord with the ethical standards of the institution's committee on human experimentation, and the protocol was reviewed and approved by the VA Boston Healthcare System Institutional Review Board. Informed consent was waived for the retrospective cohort, because the study met the Code of Federal Regulations criteria for waiver. Participants in the prospective cohort provided written informed consent. For both cohorts, patient confidentiality was protected in accordance with the policies and procedures of the US Department of Veterans Affairs. The descriptors of the retrospective ${ }^{16}$ and prospective ${ }^{17}$ cohorts have been published elsewhere.

\section{Retrospective cohort}

The retrospective cohort included veterans admitted to a tertiary VA hospital with a palliative care consult. ${ }^{16}$ Patients were excluded if use of antipsychotics prior to admission was documented within the patient's electronic medication list or in the history and physical admission note. Patients with evidence of mental status changes upon admission as documented in the admission notes were also excluded. Receipt of acute antipsychotic prescription was identified by a pharmacist utilizing point-of-care medication administration records from admission to hospital-day 14. Clinical occurrence of delirium was determined utilizing a validated chart-review instrument. The chart-review instrument has been found to have sensitivity of $74 \%$ and specificity of $83 \%$, and took 15-30 minutes per medical record to complete. ${ }^{10}$

\section{Prospective cohort}

The prospective cohort comprised veterans 65 years of age and older admitted to the tertiary VA hospital's medical ward. ${ }^{18}$ Patients were excluded if they had been admitted from a nursing home, rehabilitation center, intensive care unit, or other hospital, were expected to leave the hospital within 1 day, or had an impairment that would prevent them from completing the informed consent and cognitive screening tests. Receipt of antipsychotics was identified by a pharmacist using point-of-care medication administration records from admission to discharge. Antipsychotic use focused on new use of antipsychotics during the admission. After obtainment of informed consent, a clinical geriatrician with 10 years of delirium experience assessed patients daily for delirium. The initial delirium assessment included a comprehensive (30-45 minutes) assessment of attention, executive function, memory, and mood in conjunction with medical record review. Patients were followed daily by the expert for delirium. Delirium was diagnosed according to the diagnostic criteria of the DSM-IV-TR. ${ }^{19}$

\section{Power calculation}

Using the methodology described in the study by Bujang and Adnan, ${ }^{20}$ we estimated the prevalence of delirium at $30 \%$. With a null specificity of $50 \%$ and an antipsychotic-use specificity of $90 \%$, our analysis would be powered to detect a significant difference (power $>0.8$ ) with 17 participants and five cases with delirium.

\section{Analysis}

For the retrospective and prospective cohorts, this analysis compared the acute prescription of an antipsychotic to the diagnosis of delirium in each cohort. Standard methods were used for calculation of sensitivity, specificity, positive and negative predictive values, and likelihood ratios. Our study was not powered to detect sensitivity. 


\section{Results}

Patients within the retrospective cohort were mostly male (97\%) and older (mean age $74 \pm 13$ years). Of 217 patients analyzed, $31 \%(\mathrm{n}=67)$ developed delirium. Table 1 described the populations. Delirious and nondelirious patients did not differ significantly. Within this cohort, $18 \%(\mathrm{n}=40)$ of patients were prescribed an antipsychotic during their admission. Patients within the prospective validation cohort were also mostly male (94\%) and of older age (mean age $81 \pm 7$ years). Of 100 patients analyzed, $23 \%(\mathrm{n}=23)$ developed delirium and $5 \%(n=5)$ were prescribed an antipsychotic.

The outcomes of the retrospective and prospective cohorts are described in Table 2. The use of acute antipsychotics within the retrospective cohort indicated sensitivity of $54 \%$ and specificity of $97 \%$ for delirium. This corresponded to positive predictive accuracy of $90 \%$ and negative predictive accuracy of $83 \%$. Within the prospective validation cohort, acute antipsychotic prescribing indicated delirium with sensitivity of $22 \%$ and specificity of $100 \%$. This corresponded to positive predictive accuracy of $100 \%$ and negative predictive accuracy of $81 \%$.

\section{Discussion}

The use of acute antipsychotic prescribing is a highly specific method to identify delirious patients. Low sensitivity indicates that this tool may not identify all delirious patients. Despite challenges with sensitivity, high specificity of this method enhances the ability to identify patients with delirium efficiently and correctly. This method can be expected best

Table I Retrospective and prospective cohorts

\begin{tabular}{lll}
\hline & $\begin{array}{l}\text { Retrospective } \\
\text { cohort, } \mathbf{n = 2 1 7}\end{array}$ & $\begin{array}{l}\text { Prospective } \\
\text { cohort, } \mathbf{n = 1 0 0}\end{array}$ \\
\hline Age, years (SD) & $72.9(12.8)$ & $80.8(7.4)$ \\
Male & $97 \%(210)$ & $94 \%(94)$ \\
Prior dementia diagnosis & $3 \%(8)$ & $12 \%(12)$ \\
Delirium & $31 \%(67)$ & $23 \%(23)$ \\
Antipsychotic use & $18 \%(40)$ & $5 \%(5)$ \\
\hline
\end{tabular}

Table 2 Performance of acute antipsychotic prescription as a marker of delirium

\begin{tabular}{lll}
\hline Outcome & $\begin{array}{l}\text { Retrospective } \\
\text { cohort, } \mathbf{n = 2 1 7}\end{array}$ & $\begin{array}{l}\text { Prospective } \\
\text { cohort, } \mathbf{n = 1 0 0}\end{array}$ \\
\hline Sensitivity & $54 \%$ & $22 \%$ \\
Specificity & $97 \%$ & $100 \%$ \\
Positive predictive value & $90 \%$ & $100 \%$ \\
Negative predictive value & $82 \%$ & $81 \%$ \\
Positive likelihood ratio & 20.1 & $-*$ \\
Negative likelihood ratio & 0.48 & 0.78 \\
\hline
\end{tabular}

Note: *Positive likelihood ratio did not exist, because all patients prescribed antipsychotics developed delirium. to identify those with hyperactive delirium. The benefit to a health-system or quality-improvement program is the efficiency of this mechanism to identify delirium at the point of prescribing, allowing timely, clinical efforts to be targeted to those patients who may be delirious. While not all patients with delirium will be identified, this provides a mechanism to identify a group of patients for targeted interventions.

The use of antipsychotic medications for the treatment of delirium has been debated intensely over the past decade. There are multiple systematic reviews analyzing a few small randomized trials. ${ }^{21-26}$ The available evidence limits the conclusions of these reviews. With a recent randomized controlled trial demonstrating no benefit and possible harms of antipsychotics in a palliative population, ${ }^{15}$ the findings of this study are timely and can be used to support clinical efforts.

Delirium is a difficult clinical diagnosis. Completion of prospective, standardized mental status testing followed by application of diagnostic criteria (time 20-30 minutes) yields high sensitivity (94\%) and high specificity $(89 \%){ }^{27}$ When diagnostic criteria are completed by nurses without the mental status testing (time 3-7 minutes), sensitivity decreases to $15 \%-31 \%$, while specificity remains high $-90 \%-99 \%{ }^{4}$ Brief objective screening instruments, such as the Confusion Assessment Method for intensive care-unit patients (time $<2$ minutes $)^{28}$ and modified Richmond Agitation and Sedation Scale (time 15 seconds), have been challenged by low sensitivity while maintaining high specificity. ${ }^{17}$ Retrospective case review (time 15-30 minutes) of physician and nursing notes has also been shown to be relatively insensitive, with rates of $12 \%-70 \% .^{2-5}$ The retrospective chart-review instrument utilized here (time 15-30 minutes) improves sensitivity to 74\% without much change in specificity of $83 \% .{ }^{10}$ The sensitivity and specificity of acute antipsychotic prescribing to identify delirious patients is consistent with the other instruments that lack standardized mental status assessments.

While a highly specific but insensitive measure might not capture every delirious patient, such a measure would allow for efficient, targeted identification and clinical evaluation of such patients who are likely to have delirium. Many diagnostic algorithms utilize highly specific measures to capture patients with disease. For example, chest pain with radiation has sensitivity of $38 \%$ and specificity of $91 \%$ for acute myocardial infarction. ${ }^{29} \mathrm{~A}$ simple and efficient measure, such as acute antipsychotic prescribing, could be a marker of patients who need additional evaluation for delirium. In the face of poor routine delirium diagnosis, ${ }^{30,31}$ acute antipsychotic prescribing as a measure could bring much-needed education and awareness. ${ }^{32}$ 
This analysis is limited by population characteristics. As a Veterans Affairs Medical Center, our institution cares for veterans who are predominately older adult males. This limits the generalizability of our findings to the overall aging population, which is predominately female. Future work is needed to study a more heterogeneous cohort. The retrospective cohort also focused on palliative care inpatients, a high-risk group for the development of delirium. In addition, our sample would benefit from additional descriptive data on the cause of admission; however, analysis of such cohorts would be power-limited. The communication of delirium across transitions of care was not addressed in the study protocol, but is an important clinical element of hospitalized patients with delirium. Antipsychotic medications are more frequently prescribed for hyperactive delirium, which is the minority of cases. ${ }^{33}$ Our cohorts lacked sufficient size or methods to allow accurate subanalysis of the specificity of delirium subtypes. The utility of this method to capture hypoactive delirium is a limitation. However, antipsychotic prescription improves the efficiency of identifying delirium, particularly when merged with expert clinician interview or standardized chart-review instruments. These standard methods require more time, and while more sensitive, do not offer a high level of efficiency in identifying delirium for health-system clinical or quality-improvement initiatives.

Utilization of efficient, highly specific methods to identify delirium cases is of great importance to quality-improvement specialists, researchers, and hospital systems. Use of acute antipsychotic prescription as an indicator of delirium occurrence may expedite case identification. This method is easily implemented into electronic health systems at no cost. Validation of this method in other population cohorts may be warranted to expand applicability, and interventions would require additional assessment.

In retrospective and prospective cohorts, we found prescription of a new antipsychotic during hospitalization to be very specific, but relatively insensitive to clinical occurrence of delirium. While the method does not capture all delirious patients, those who receive new antipsychotic prescriptions are likely to be delirious. This method could be embedded into existing electronic health-information systems within hospital settings to identify delirious cases. The utilization of this quick and inexpensive identification method may expand case identification for research and clinical interventions.

\section{Clinical implications}

Inpatients prescribed antipsychotics are likely to have delirium, but not all patients with delirium receive antipsychotics. Within a health system, antipsychotic prescription could trigger targeted clinical intervention. Quality-improvement methods could use antipsychotic prescription to gauge the impact of nonpharmacological measures for delirium.

\section{Acknowledgments}

This work was funded by a VA Delirium Patient Safety Center of Inquiry, a VA T21 alternative to noninstitutional long-term care grant, and NIH 1T35AG038027-01. Additional support was provided by the ASHP Foundation Award for Clinical Excellence in Medication Use Safety (2012). We are thankful to the veterans who participated. We are appreciative of Ms Kelly Doherty's and Ms Dawn Mello's effort in editing and proofreading the manuscript. The views expressed in this article are those of the authors and do not necessarily reflect the position or policy of the Department of Veterans Affairs or the United States Government. The abstract of this paper was presented at the American Society of Health-System Pharmacists 2013 Midyear Clinical Meeting. The poster's abstract was published in the meeting program, which can be found at: https://www.ashp.org/meetings-and-events/meetingsand-conferences/midyear-clinical-meeting-and-exhibition/ midyear-clinical-meeting-abstracts-archive.

\section{Disclosure}

JLR is supported by the VA Health Services Research and Development Center of Innovation in Long-Term Services and Supports (CIN 13-419) and the VA Quality Enhancement Research Initiative Geriatrics and Extended Care Partnered Evaluation Center for Community Nursing Homes (PEC 15-465). JLR is a US Government employee. The authors report no other conflicts of interest in this work.

\section{References}

1. Inouye SK. Delirium in older persons. $N$ Engl J Med. 2006;354: 1157-1165.

2. Siddiqi N, House AO, Holmes JD. Occurrence and outcome of delirium in medical in-patients: a systematic literature review. Age Ageing. 2006;35:350-364.

3. Hosie A, Davidson PM, Agar M, Sanderson CR, Phillips J. Delirium prevalence, incidence, and implications for screening in specialist palliative care inpatient settings: a systematic review. Palliat Med. 2013;27:486-498.

4. Inouye SK, Foreman MD, Mion LC, Katz KH, Cooney LM Jr. Nurses' recognition of delirium and its symptoms: comparison of nurse and researcher ratings. Arch Intern Med. 2001;161:2467-2473.

5. van Eijk MM, van Marum RJ, Klijn IA, de Wit N, Kesecioglu J, Slooter AJ. Comparison of delirium assessment tools in a mixed intensive care unit. Crit Care Med. 2009;37:1881-1885.

6. Han JH, Zimmerman EE, Cutler N, et al. Delirium in older emergency department patients: recognition, risk factors, and psychomotor subtypes. Acad Emerg Med. 2009;16:193-200.

7. Rice KL, Bennett MJ, Clesi T, Linville L. Mixed-methods approach to understanding nurses' clinical reasoning in recognizing delirium in hospitalized older adults. J Contin Educ Nurs. 2014;45:136-148. 
8. Marcantonio ER, Ngo LH, O'Connor M, et al. 3D-CAM: derivation and validation of a 3-minute diagnostic interview for CAM-defined delirium: a cross-sectional diagnostic test study. Ann Intern Med. 2014;161:554-561.

9. Johnson JC, Kerse NM, Gottlieb G, Wanich C, Sullivan E, Chen K. Prospective versus retrospective methods of identifying patients with delirium. J Am Geriatr Soc. 1992;40:316-319.

10. Inouye SK, Leo-Summers L, Zhang Y, Bogardus ST Jr, Leslie DL, Agostini JV. A chart-based method for identification of delirium: validation compared with interviewer ratings using the Confusion Assessment Method. J Am Geriatr Soc. 2005;53:312-318.

11. National Institute for Health and Care Excellence. Delirium: Prevention, Diagnosis and Management. London: NICE; 2010.

12. Bush SH, Leonard MM, Agar M, et al. End-of-life delirium: issues regarding recognition, optimal management, and the role of sedation in the dying phase. J Pain Symptom Manage. 2014;48:215-230.

13. Fok MC, Sepehry AA, Frisch L, et al. Do antipsychotics prevent postoperative delirium? A systematic review and meta-analysis. Int J Geriatr Psychiatry. 2015;30:333-344.

14. Breitbart W, Alici-Evcimen Y. Why off-label antipsychotics remain first-choice drugs for delirium. Curr Psychiatry. 2010;6:49-63.

15. Agar MR, Lawlor PG, Quinn S, et al. Efficacy of oral risperidone, haloperidol, or placebo for symptoms of delirium among patients in palliative care: a randomized clinical trial. JAMA Intern Med. 2017; 177:34-42.

16. Zimmerman KM, Salow M, Skarf LM, et al. Increasing anticholinergic burden and delirium in palliative care inpatients. Palliat Med. 2014;28:335-341.

17. Chester JG, Harrington MB, Rudolph JL. Serial administration of a modified Richmond Agitation and Sedation Scale for delirium screening. J Hosp Med. 2012;7:450-453.

18. Rudolph JL, Harrington MB, Lucatorto MA, Chester JG, Francis J, Shay KJ. Validation of a medical record-based delirium risk assessment. J Am Geriatr Soc. 2011;59 Suppl 2:S289-S294.

19. American Psychiatric Association. Diagnostic and Statistical Manual of Mental Disorders. 4th ed. Washington: APA; 2000.

20. Bujang MA, Adnan TH. Requirements for minimum sample size for sensitivity and specificity analysis. J Clin Diagn Res. 2016; $10: 1-6$.
21. Lacasse H, Perreault MM, Williamson DR. Systematic review of antipsychotics for the treatment of hospital-associated delirium in medically or surgically ill patients. Ann Pharmacother. 2006;40:1966-1973.

22. Seitz DP, Gill SS, van Zyl LT. Antipsychotics in the treatment of delirium: a systematic review. J Clin Psychiatry. 2007;68:11-21.

23. Markowitz JD, Narasimhan M. Delirium and antipsychotics: a systematic review of epidemiology and somatic treatment options. Psychiatry (Edgmont). 2008;5:29-36.

24. Flaherty JH, Gonzales JP, Dong B. Antipsychotics in the treatment of delirium in older hospitalized adults: a systematic review. J Am Geriatr Soc. 2011;59 Suppl 2:S269-S276.

25. Kishi T, Hirota T, Matsunaga S, Iwata N. Antipsychotic medications for the treatment of delirium: a systematic review and meta-analysis of randomised controlled trials. J Neurol Neurosurg Psychiatry. 2016;87:767-774.

26. Neufeld KJ, Yue J, Robinson TN, Inouye SK, Needham DM. Antipsychotic medication for prevention and treatment of delirium in hospitalized adults: a systematic review and meta-analysis. J Am Geriatr Soc. 2016;64:705-714.

27. Wei LA, Fearing MA, Sternberg EJ, Inouye SK. The confusion assessment method: a systematic review of current usage. J Am Geriatr Soc. 2008;56:823-830.

28. Neufeld KJ, Leoutsakos JS, Sieber FE, et al. Evaluation of two delirium screening tools for detecting post-operative delirium in the elderly. $\mathrm{Br}$ J Anaesth. 2013;111:612-618.

29. Goodacre S, Locker T, Morris F, Campbell S. How useful are clinical features in the diagnosis of acute, undifferentiated chest pain? Acad Emerg Med. 2002;9:203-208.

30. Hope C, Estrada N, Weir C, Teng CC, Damal K, Sauer BC. Documentation of delirium in the VA electronic health record. BMC Res Notes. 2014;7:208

31. Hustey FM, Meldon SW. The prevalence and documentation of impaired mental status in elderly emergency department patients. Ann Emerg Med. 2002;39:248-253.

32. Rudolph JL, Boustani M, Kamholz B, Shaughnessey M, Shay K. Delirium: a strategic plan to bring an ancient disease into the $21 \mathrm{st}$ century. J Am Geriatr Soc. 2011;59 Suppl 2:S237-S240.

33. Yang FM, Marcantonio ER, Inouye SK, et al. Phenomenological subtypes of delirium in older persons: patterns, prevalence, and prognosis. Psychosomatics. 2009;50:248-254.
Clinical Pharmacology: Advances and Applications

\section{Publish your work in this journal}

Clinical Pharmacology: Advances and Applications is an international, peer-reviewed, open access journal publishing original research, reports, reviews and commentaries on all areas of drug experience in humans. The manuscript management system is completely online and includes a very quick and fair peer-review system, which is all easy to use

\section{Dovepress}

Visit http://www.dovepress.com/testimonials.php to read real quotes from published authors. 